\title{
Determinantes de la Relación Entre el Precio y el Valor Contable de las Acciones
}

\section{Manuel García-Ayuso Covarsí \& Juan Antonio Rueda Torres}

To cite this article: Manuel García-Ayuso Covarsí \& Juan Antonio Rueda Torres (2002)

Determinantes de la Relación Entre el Precio y el Valor Contable de las Acciones, Spanish Journal of Finance and Accounting / Revista Española de Financiación y Contabilidad, 31:114, 1013-1039, DOI: $10.1080 / 02102412.2002 .10779467$

To link to this article: https://doi.org/10.1080/02102412.2002.10779467

\section{Published online: 15 Jan 2014.}

Submit your article to this journal $\widetilde{3}$

Џll Article views: 37 


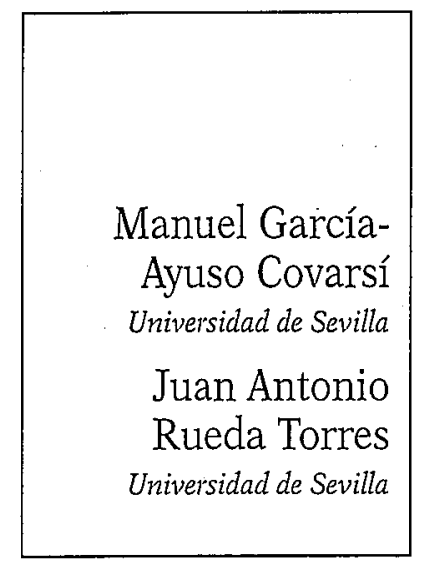

\section{DETERMINANTES DE LA RELACIÓN ENTRE EL PRECIO Y EL VALOR CONTABLE DE LAS ACCIONES $(*)$}

Resumen._Palabras clave._Abstract.-Key words.-1. Introducción.-

2. Muestra e hipótesis contrastadas: 2.1. Muestra analizada. 2.2. Hipótesis de partida.-3. Evidencia empirica: 3.1. Análisis de correlación. 3.2. Análisis de regresión-4. Resumen y consideraciones finales.-Referencias bibliográficas.

\section{RESUMEN}

$\mathrm{L}$

A teoría financiera predice que la relación entre el precio y el valor contable de las acciones (el ratio PB) será tanto mayor cuanto mayor sean las expectativas de rentabilidad y crecimiento de los recursos propios, y cuanto menor sea la rentabilidad exigida por los accionistas. Este trabajo contrasta estas relaciones a partir de una muestra de

(*) Agradecemos al Servicio de Estudios de la Bolsa de Madrid su inestimable ayrida al hacer accesible parte de la información necesaria para la elaboración de la base de datos empleada en esta investigación. Asimismo, estamos agradecidos a Juan Carlos Gómez Sala y a los asistentes al Encuentro de ASEPUC celebrado en Las Palmas, por los comentarios recibidos sobre una versión preliminar de este estudio. Este trabâjo es un resultado del proyecto de investigación PB98-0415, financido por la Dirección General de Investigación Científica y Técnica del Ministerio de Educación y Ciencia. 
empresas cotizadas en la Bolsa de Madrid durante el período 1986-2000. Nuestros resultados confirman la existencia de una relación positiva entre los valores contemporáneos del ratio $\mathrm{PB}$ y la rentabilidad financiera de las empresas. Al mismo tiempo, la rentabilidad financiera muestra una elevada persistencia, que se traduce en una relación positiva entre el ratio $\mathrm{PB}$ actual y las rentabilidades financieras futuras. Sin embargo, las rentabilidades observadas en cada año permiten predecir las rentabilidades a corto plazo mejor que el propio ratio $\mathrm{PB}$, mientras que a muy largo plazo el ratio $\mathrm{PB}$ es una variable predictiva mejor de las rentabilidades. Por último, también constatamos una relación positiva del ratio $\mathrm{PB}$ con el crecimiento futuro de los recursos propios, y negativa con respecto al riesgo medido por el coeficiente $\beta$ del modelo de mercado.

\section{PALABRAS CLAVE}

Valoración de activos; Mercados de capitales; Ratio precio-valor contable; Rentabilidad financiera; Crecimiento.

\section{ABSTRACT}

In a efficient market, financial theory predicts that price-to-book ( $P B)$ ratios should depend on expected future return on equity (ROE), the related growth in book value of equity, and the required rate of return. This paper presents the results of an empirical analysis of those relations using a sample of firms listed in the Madrid stock exchange during the period 1986-2000. The evidence presented here is consistent with the results of previous studies in that it suggests that current $P B$ and ROE are positively correlated. Additionally, the persistence in ROE drives a positive relationship between the $\mathrm{PB}$ ratio and the future ROEs, and current $\mathrm{PB}$ ratio have explanatory power for future ROEs in the long term beyond current ROE. Finally, we found a positive relationship between the $\mathrm{PB}$ ratio and the future growth in book values, and a negative relationship between the $\mathrm{PB}$ ratio and the risk when it is measured using the $\beta$ coefficient of the market model. 


\section{KEY WORDS}

Asset pricing; Capital markets; Price-to-book ratio; Return on equity; Growth.

\section{INTRODUCCIÓN}

La relación entre el precio y el valor contable de las acciones (ratio PB en lo sucesivo) es, junto con la relación entre el precio y los resultados, una de las variables que mayor atención ha recibido por parte de profesionales y académicos en la evaluación y selección de inversiones financieras. En este sentido, la investigación empírica ha documentado de manera reiterada una relación significativamente negativa entre el ratio PB y la rentabilidad media de las acciones negociadas en los mercados de Estados Unidos [Rosenberg et al., 1985; Fama y French, 1992; Jensen et al., 1997], Japón [Chan et al., 1991], Reino Unido [Chan y Chui, 1996], España [Rueda, 2001] y otra serie de países [Capaul et al., 1993; Fama y French, 1998].

Cuando se controlan las diferencias en el riesgo de los títulos, medido generalmente por el coeficiente $\beta$ del CAPM, estos trabajos también han constatado la posibilidad de realizar una rentabilidad anormal significativa utilizando una estrategia consistente en la inversión de los títulos que muestran unos valores más bajos del ratio $\mathrm{PB}$, financiada con la venta (al descubierto o a crédito) de aquellos otros que muestran un valor más alto de ese ratio. Como explicación de esta anomalía, se ha argumentado que los valores relativamente altos (bajos) del ratio $\mathrm{PB}$ identifican empresas temporalmente sobrevaloradas (infravaloradas), debido a que las expectativas de los inversores acerca de su capacidad de generación de riqueza son excesivamente optimistas (pesimistas). Esta interpretación del efecto $P B$ es avalada, entre otros, por los trabajos de Farfield y Harris [1993], Lakonishok et al. [1994] y La Porta et al. [1997]. En cambio, Fama y French $[1995,1996]$ sugieren que el efecto $P B$ puede ser consistente con la eficiencia del mercado y obedecer a una especificación errónea del CAPM, de manera que los valores altos (bajos) del ratio PB identifican títulos cuyo riesgo sistemático es inferior (superior) al que predicen sus respectivos coeficientes $\beta$ (1).

(1) Por su parte, Kothari et al. [1994] demuestran que el efecto $P B$ es una regularidad espúrea, inducida por la muestra analizada y el método de estudio utilizado en su contrastación, lo cual sin embargo es refutado por Davis [1994] y Chan et al. [1995]. 
Ahora bien, la solución al debate sobre si la variación del ratio PB refleje o no un sesgo en la valoración de los activos requiere asumir a priori un modelo que especifique cuál es el valor de equilibrio (o intrínseco) del ratio $\mathrm{PB}$, observable para cada empresa bajo condiciones de eficiencia del mercado. De esta forma, cabría contrastar si una estrategia sobre los títulos con un ratio PB muy bajo y muy alto en relación con el valor que predice ese modelo permite obtener una rentabilidad anormal significativa. En este sentido, el modelo de Ohlson [1995] ofrece una caracterización del ratio $\mathrm{PB}$ como función de las expectativas de rentabilidad financiera futura, el crecimiento de los recursos propios y la rentabilidad exigida por los inversores.

Numerosos estudios empíricos han contrastado la validez de esta caracterización, como paso previo a su utilización en la selección de inversiones. Así, Penman [1991] documenta que el ratio PB permite predecir las rentabilidades financieras futuras, incorporando alguna información que no es reflejada por la contabilidad en la rentabilidad financiera actual. Fama y French [1995] también documentan una relación negativa entre el inverso del ratio $\mathrm{PB}$ y las rentabilidades pasadas y futuras, que persiste cuando se controlan las diferencias en la capitalización de las empresas, y Penman [1996] constata una relación positiva de las rentabilidades futuras con el ratio $\mathrm{PB}$ actual cuando aquéllas se ajustan por una estimación del coste de capital (idéntica para todas las empresas).

No obstante, la evidencia hallada por Bernard [1994] indica que las diferencias en la rentabilidad financiera y el crecimiento futuro de los recursos propios sólo pueden explicar un fracción reducida de la variación actual del ratio PB. De hecho, la rentabilidad financiera de las empresas con un ratio $\mathrm{PB}$ más bajo en el año actual tiende a mantenerse en sensiblemente invariable a lo largo de los años siguientes, y es muy similar a la rentabilidad obtenida sobre sus recursos propios por las empresas con un ratio $\mathrm{PB}$ más alto.

Sin abandonar la hipótesis de eficiencia del mercado, ello puede obedecer a la existencia de un sesgo conservador (agresivo) persistente en la cifras contables de resultados y recursos propios de las empresas que muestran un ratio PB más alto (bajo), que impedirá observar una reversión a la media de las rentabilidades de esas empresas, aun cuando se alcance una situación de equilibrio competitivo. En este sentido, los resultados aportados por Ryan [1995] y Beaver y Ryan [2000] son consistentes con el hecho de que el principio de coste histórico se traduce en un valoración contable más conservadora del patrimonio neto (y en un ratio $\mathrm{PB}$ más alto, ceteris paribus) de las empresas intensivas en capital fijo. Por 
su parte, Lev y Sougiannis [1999] también constatan que la contabilización como gasto del capital invertido en las actividades de investigación y desarrollo motiva la observación de un ratio $\mathrm{PB}$ más alto para las empresas intensivas en tales actividades.

El objeto de este trabajo es contrastar la relación del ratio PB con sus determinantes fundamentales sobre una muestra de empresas negociadas en el mercado de capitales español a lo largo del período 1986-2000. La evidencia hallada confirma la existencia de una relación positiva entre los valores actuales del ratio PB y la rentabilidad financiera de las empresas. Por su parte, las diferencias entre la rentabilidad financiera actual de las empresas se mantienen de forma persistente a largo plazo, lo que se traduce en una relación positiva entre el ratio PB y las rentabilidades financieras futuras. Asimismo, los valores actuales del ratio PB actual permiten pronosticar las rentabilidades a muy largo plazo mejor que la rentabilidad actual. Por último, nuestros resultados también pone de manifiesto una relación positiva del ratio $\mathrm{PB}$ con el crecimiento futuro de los recursos propios, y negativa con respecto al riesgo medido por el coeficiente $\beta$ del modelo de mercado.

El resto del trabajo se estructura en tres partes diferenciadas. En el epígrafe siguiente se describen los datos utilizados en la investigación empírica, así como el modelo teórico que especifica la relación del ratio $\mathrm{PB}$ con sus determinantes fundamentales. El epígrafe tercero presenta los resultados de la investigación, utilizando un análisis de correlación y de regresión entre el ratio $\mathrm{PB}$ y los factores que predice la teoría financiera. Por último, en el epígrafe cuarto se resumen los resultados del trabajo y se apuntan algunas futuras líneas de investigación.

\section{MUESTRA E HIPÓTESIS CONTRASTADAS}

\subsection{Muestra ANALIzADA}

Para la realización del estudio empírico utilizamos una base de datos que recopila información de 177 empresas admitidas a negociación en la Bolsa de Madrid durante el periodo 1985-2000. La información disponible para cada empresa es la siguiente:

- Cifras de recursos propios y resultado ordinario correspondientes a uno o varios ejercicios consecutivos cerrados durante el período referido. La cifra de recursos propios comprende el capital social, las reservas y el beneficio neto del ejercicio, siendo deducidos los 
dividendos entregados a cuenta. La cifra de resultados excluye los componentes de naturaleza extraordinaria y el gasto por impuestos. Ambos datos fueron recopilados del Sistema de Información Bursátil hasta el año 1990, y de los registros de la Comisión Nacional del Mercado de Valores en los años siguientes. Asimismo, se optó por utilizar los estados financieros consolidados desde que resultaron disponibles.

- Precio de las acciones al cierre de la última sesión de cada ejercicio y número total de títulos admitidos a cotización en esa fecha, que fueron recopilados a partir de los Boletines Oficiales de Cotización de la Bolsa de Madrid.

- Rentabilidades mensuales de las acciones desde enero de 1986 hasta diciembre del 2000. Estas rentabilidades se calcularon ajustando la variación relativa del precio por los dividendos, ampliaciones de capital y desdobles del nominal de los títulos (splits).

Utilizando esta información, calculamos las siguientes variables para cada empresa y año:

- Ratio precio-valor contable, $P B$, resultante de dividir el precio de las acciones observado al término de cada ejercicio entre su valor contable ajustado por los splits.

- Rentabilidad financiera, $R O E$, calculada como el cociente del resultado ordinario del ejercicio cerrado en el año $t$ entre el valor contable de los recursos propios al inicio de ese ejercicio. Tanto esta variable como el ratio PB sólo se computan cuando el denominador es positivo.

- Tasa de crecimiento de los recursos propios (ajustado por los splits), g.

- Rentabilidad bursátil anual, $R$, que se obtiene capitalizando las rentabilidades mensuales observadas durante los 12 meses del ejercicio $t$.

La tabla 1 refleja el valor mediano del ratio PB y el valor medio de las restantes variables en cada año del período 1986-2000. La utilización de la mediana del ratio PB reduce el efecto de las observaciones extremas, dada la dispersión que caracteriza la distribución de esta variable. En las dos últimas filas de la tabla 1 figuran la media aritmética y geométrica de cada variable sobre los 15 años. Puede apreciarse que la media geométrica muestra un valor inferior al de la media aritmética, especialmente en el caso de la rentabilidad bursátil que manifiesta una mayor volatilidad. 
TABLA 1

RATIO PB, CRECIMIENTO Y RENTABILIDAD FINANCIERA Y BURSÁTIL ANUAL (PERÍODO 1986-2000)

\begin{tabular}{|c|c|c|c|c|r|}
\hline Año & $\begin{array}{c}N .^{\circ} \\
\text { titulos }\end{array}$ & $\begin{array}{c}P B \\
\text { mediano }\end{array}$ & $\begin{array}{c}g \\
\text { medio }\end{array}$ & $\begin{array}{c}\text { ROE } \\
\text { medio }\end{array}$ & $\begin{array}{r}R \\
\text { medio }\end{array}$ \\
\hline 1986 & 52 & 1,139 & 0,231 & 0,113 & 1,110 \\
1987 & 54 & 1,192 & 0,073 & 0,108 & 0,225 \\
1988 & 62 & 1,343 & 0,242 & 0,122 & 0,165 \\
1989 & 75 & 1,630 & 0,021 & 0,165 & $-0,009$ \\
1990 & 87 & 0,932 & 0,081 & 0,116 & $-0,038$ \\
1991 & 88 & 0,895 & 0,016 & 0,065 & $-0,198$ \\
1992 & 89 & 0,667 & $-0,101$ & $-0,019$ & $-0,248$ \\
1993 & 90 & 1,254 & $-0,059$ & $-0,112$ & 0,647 \\
1994 & 95 & 1,223 & $-0,001$ & 0,030 & $-0,114$ \\
1995 & 96 & 1,122 & 0,185 & 0,152 & 0,236 \\
1996 & 102 & 1,260 & 0,209 & 0,144 & 0,379 \\
1997 & 107 & 1,633 & 0,113 & 0,174 & 0,961 \\
1998 & 121 & 1,629 & 0,080 & 0,212 & $-0,048$ \\
1999 & 125 & 1,037 & 0,275 & 0,223 & 0,034 \\
2000 & 120 & 0,924 & 0,125 & 0,217 & $-0,084$ \\
\hline Media aritmética & 1,192 & 0,099 & 0,114 & 0,201 \\
Media geométrica & & 0,094 & 0,110 & 0,145 \\
\hline
\end{tabular}

El ratio $\mathrm{PB}$ es la relación entre el precio y el valor contable por acción (ajustado por splits) observado al término del ejercicio cerrado en cada año. La tasa $g$ mide el crecimiento de los recursos propios (ajustado por splits) en cada año. La tasa ROE es la rentabilidad financiera, igual al cociente del resultado ordinario de cada ejercicio entre el valor contable inicial de los recursos propios. La tasa $\mathrm{R}$ es la rentabilidad bursátil anual, que se obtiene capitalizando las rentabilidades mensuales observadas durante los 12 meses de cada ejercicio.

A la vista de esta tabla, se aprecia que el ratio PB muestra a lo largo del período analizado un valor medio que es ligeramente superior a la unidad, y la rentabilidad financiera (11\%) es inferior a la rentabilidad bursátil (14,5\%). Ambas características son consistentes con un sesgo conservador de las cifras contables de resultados y recursos propios en el reflejo de la información que es incorporada por el mercado a los precios.

También se puede apreciar que el ratio $\mathrm{PB}$ alcanza sus valores máximos en los años 1989 y 1997, observándose entre estos dos años un declive y posterior recuperación de la relación entre el valor de mercado y el valor contable de las empresas. La figura 1 pone de manifiesto una co- 


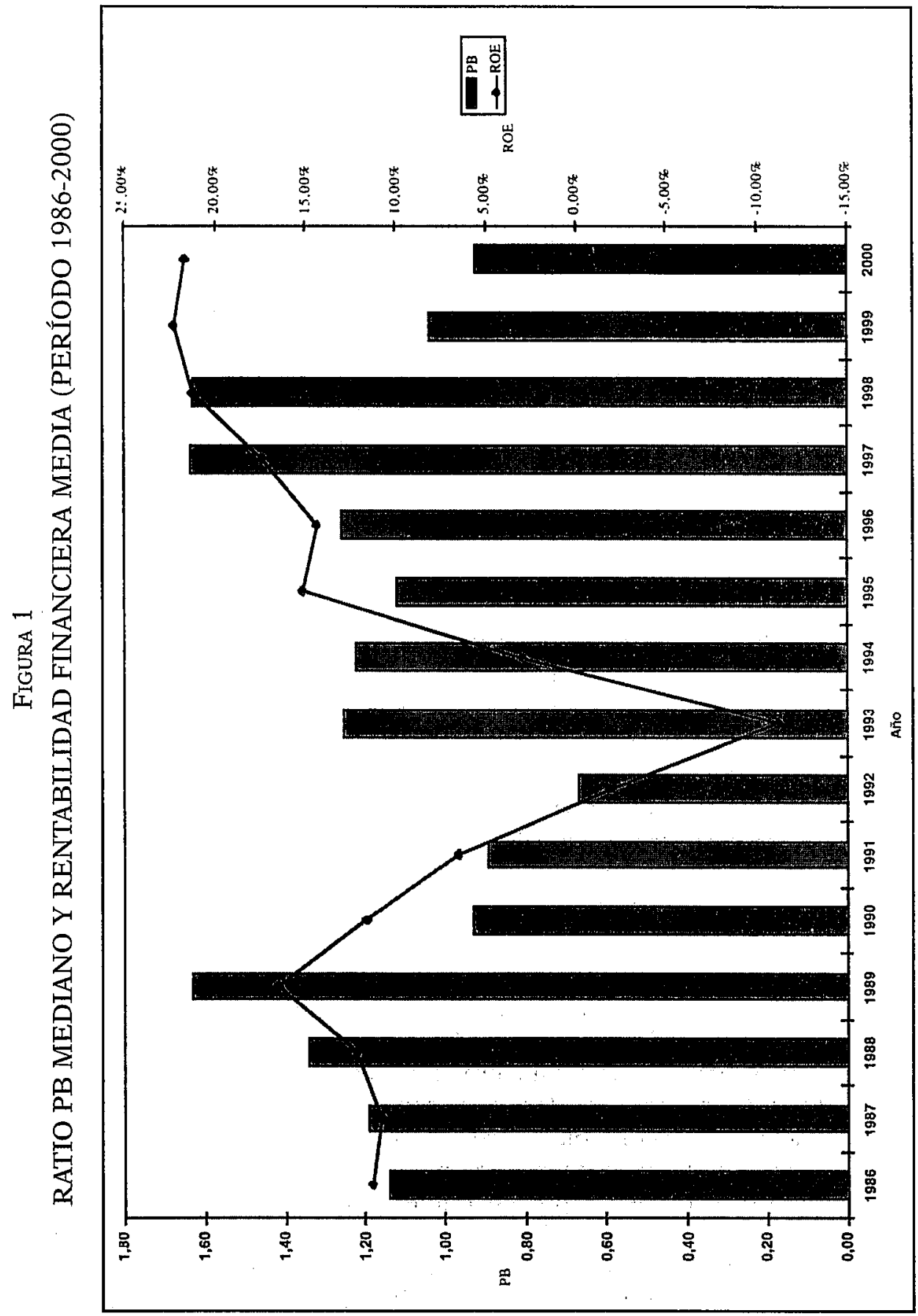


rrespondencia sustancial entre la tendencia seguida por el ratio PB y la rentabilidad financiera para el conjunto del mercado a lo largo del período analizado.

El valor mínimo del ratio $\mathrm{PB}$, registrado en 1992, coincide con el mayor declive experimentado por el precio de los títulos (la rentabilidad media realizada en ese año es igual a un -25\%), así como con un decrecimiento de los recursos propios y una rentabilidad financiera negativa. Por su parte, el máximo alcanzado por el ratio PB en 1997 está motivado por la tendencia al alza experimentada por los precios en ese año, la cual parece anticipar el incremento en la rentabilidad obtenida por las empresas sobre sus recursos propios, que alcanza sus valores máximos en los tres últimos años del período analizado.

A partir del panel de observaciones empresa-año disponibles, se descartaron:

- Las empresas financieras (bancos, sociedades de seguro y de inversión), consistentemente con la práctica habitual seguida en estudios previos. Ello obedece a la peculiaridad de los estados contables de esas empresas los hace difícilmente comparables con los publicados por las empresas no financieras.

- Las observaciones extremas de PB, ROE y $g$. A tal efecto, decidimos excluir los valores de $\mathrm{PB}$ superiores a 8 , así como las tasas de rentabilidad y crecimiento superiores a un $100 \%$ en valor absoluto.

- Las empresas para las que en cada año no se dispone de su rentabilidad financiera y/o el crecimiento de sus recursos propios en el año siguiente. Ello motivó la eliminación de las observaciones del año 2000.

La muestra resultante, utilizada en el estudio posterior, quedó integrada por 127 empresas, para las que se dispone de un total de 997 observaciones empresa-año a lo largo del período 1986-1999. El número de observaciones en cada año oscila entre un mínimo de 41 para 1986 y un máximo de 85 en 1999.

\subsection{HIPÓTESIS DE PARTIDA}

A partir del modelo de valoración de acciones basado en el descuento de los dividendos futuros, Ohlson [1995] demuestra que, bajo condiciones de equilibrio, el valor de los recursos propios de una empresa se puede expresar como la suma de su valor actual en libros más el valor actual de las expectativas del mercado acerca de sus futuros resultados anormales (en 
exceso sobre la remuneración exigida por los accionistas). A su vez, este modelo de valoración permite deducir la siguiente expresión del ratio PB propuesta por Bernard [1994]:

$$
\frac{P_{1}}{B_{t}}=1+\sum_{\tau=1}^{\infty}(1+r)^{-t}\left[\left(R O E_{t+\tau}-r\right) \frac{B_{t+\tau-1}}{B_{t}}\right]
$$

donde: $P$, es el valor de mercado de los recursos propios en el momento $t$; $B_{1}$ es su valor contable, y $r$ es la tasa de rentabilidad exigida por los inversores (o coste de los recursos propios), que se supone constante en el tiempo.

De acuerdo con el modelo [1], un valor del ratio PB superior (inferior) a la unidad se explica por una expectativa de rentabilidad superior (inferior) a la remuneración exigida por los inversores. A su vez, el crecimiento esperado de los recursos propios ejerce un efecto palanca, al amplificar o minimizar el efecto de la rentabilidad anormal esperada (positiva o negativa) sobre el ratio $\mathrm{PB}$. Por último, manteniendo el resto de los factores constantes, cuanto menor sea la rentabilidad exigida por los inversores (en función del riesgo asumido) mayor será el ratio $\mathrm{PB}$.

La contrastación del modelo [1] suscita el problema de que las expectativas del mercado no son directamente observables. Consiguientemente, en nuestra investigación asumiremos que las observaciones ex post de la rentabilidad financiera y el crecimiento de los recursos propios proporcionan una buena medida de las expectativas ex ante cuando se promedian sobre muestras suficientemente grandes y, asimismo, que la diferencia en la rentabilidad exigida a cada empresa es función de su coeficiente beta estimado a partir del modelo de mercado.

Por tanto, las tres hipótesis que contrastamos se pueden formalizar del siguiente modo:

$H_{1}$ : El ratio PB ésta relacionado positivamente con la rentabilidad financiera futura.

$\mathrm{H}_{2}$ : La relación positiva del ratio $\mathrm{PB}$ con la rentabilidad financiera futura será más acentuada cuanto mayor sea el crecimiento futuro de los recursos propios.

$H_{3}$ : El ratio PB ésta relacionado negativamente con el coeficiente beta. Adicionalmente, si el mercado es eficiente e incorpora en el precio de las acciones (observado al término de cada ejercicio) otra información distinta de la reflejada por la contabilidad en la cifra de resultados, planteamos una cuarta hipótesis:

$\mathrm{H}_{4}$ : La variación actual del ratio PB explica las diferencias en las rentabilidades financieras futuras mejor que la variación de la rentabilidad financiera actual. 


\section{EVIDENCIA EMPÍRICA}

\subsection{ANÁLISIS DE CORRELACIÓN}

De manera preliminar, la contrastación de las hipótesis planteadas se lleva a cabo utilizando un análisis de la correlación entre el ratio PB y cada uno de sus determinantes fundamentales.

A tal efecto, el método de estudio seguido para analizar la relación del ratio PB con la rentabilidad financiera futura se articula en tres fases:

1. Al término de cada año $t$ del período 1986-1999 los títulos disponibles en la muestra se ordenan de acuerdo con sus valores relativos del ratio $\mathrm{PB}$ y se agrupan en diez carteras de aproximadamente igual número de títulos. La composición de las carteras formadas en cada año $t$ se mantiene en los cinco años siguientes, eliminando únicamente las empresas que desparecen de la muestra.

2. En segundo lugar, calculamos los valores medianos del ratio PB y de la rentabilidad financiera para cada una de las carteras en el año corriente $t$ y en çada año $t+\tau(\tau=1,2, \ldots, 5)(2)$. La utilización de los valores medianos por carteras de las variables estudiadas, en lugar de valores medios, se justifica para atenuar la influencia de las observaciones extremas. De igual modo, calculamos los coeficientes de correlación entre los rangos de: (i) el ratio PB mediano de las carteras en el año de formación de las mismas, y (ii) la rentabilidad financiera mediana de las carteras, tanto en el año de formación como en cada uno de los (hasta cinco) años siguientes (3). Este coeficiente de correlación también se computa utilizando las observaciones de los títulos individuales.

3. Por último, con los valores medianos por carteras tanto del ratio PB como de la rentabilidad financiera calculamos una media para cada año $t$ de formación de las carteras y cada año $t+\tau$ siguiente. Asimismo, calculamos una media de los coeficientes de correla-

(2) La limitación a cinco años del horizonte analizado obedece a que el número de empresas incluidas en cada cartera en el año $t$ tenderá a reducirse progresivamente en los años siguientes (a medida que algunas de esas empresas desaparecen de la muestra). Asimismo, añadiendo más años hacia delante se reduce el número de observaciones medianas disponibles por cartera, lo que resta fiabilidad a la media calculada con las mismas (así, por ejemplo, para $t+6$ sólo se cuenta con 9 observaciones por cartera).

(3) El coeficiente de correlación de rangos (o de Spearman) es un estadístico no paramétrico, que no exige asumir una relación lineal entre las variables analizadas ni su distribución normal. 
ción observados entre el ratio PB y la rentabilidad financiera en el año de formación y en cada uno de los cinco años siguientes. En ambos casos, el número de observaciones disponibles para calcular esas medias oscila entre un máximo de 14 para el año $t+1$ y un mínimo de 10 para $t+5$.

Los resultados del método descrito figuran en la tabla 2. El análisis de esta tabla refleja que las empresas que muestran una relación más alta entre el valor de mercado y el valor contable de sus recursos propios en

TABLA 2

\section{RENTABILIDAD FINANCIERA FUTURA DE LAS CARTERAS FORMADAS SEGÚN LA DISTRIBUCIÓN DEL RATIO PB EN CADA AÑO}

\begin{tabular}{|c|c|c|c|c|c|c|c|}
\hline Cartera & $P B_{t}$ & $R O E_{t}$ & $R O E_{t+1}$ & $R O E_{i+2}$ & $R O E_{i+3}$ & $R O E_{i+4}$ & $R O E_{t+5}$ \\
\hline 1 & 0,344 & 0,003 & 0,001 & 0,029 & 0,037 & 0,051 & 0,072 \\
2 & 0,567 & 0,034 & 0,029 & 0,031 & 0,051 & 0,083 & 0,097 \\
3 & 0,732 & 0,095 & 0,096 & 0,064 & 0,059 & 0,047 & 0,076 \\
4 & 0,874 & 0,095 & 0,103 & 0,104 & 0,109 & 0,097 & 0,081 \\
5 & 1,061 & 0,112 & 0,098 & 0,044 & 0,031 & 0,081 & 0,101 \\
6 & 1,224 & 0,148 & 0,129 & 0,150 & 0,129 & 0,140 & 0,157 \\
7 & 1,444 & 0,118 & 0,126 & 0,140 & 0,152 & 0,127 & 0,128 \\
8 & 1,721 & 0,161 & 0,158 & 0,148 & 0,146 & 0,140 & 0,165 \\
9 & 2,182 & 0,203 & 0,184 & 0,173 & 0,179 & 0,160 & 0,129 \\
10 & 3,457 & 0,239 & 0,233 & 0,241 & 0,227 & 0,207 & 0,222 \\
\hline $10-1$ & 3,113 & 0,236 & 0,233 & 0,212 & 0,190 & 0,157 & 0,150 \\
$9-2$ & 1,615 & 0,169 & 0,155 & 0,142 & 0,128 & 0,077 & 0,032 \\
\hline \multicolumn{2}{|c|}{ Correlación rangos: } & $0,769 * *$ & $0,799 * * *$ & $0,770 * *$ & $0,791 * *$ & $0,592 *$ & $0,576 *$ \\
Carteras & 0,502 & 0,493 & 0,456 & 0,472 & 0,411 & 0,372 \\
\hline
\end{tabular}

Al término de cada año $t(t=1986, \ldots, 1999)$ se ordenan los títulos según su ratio $\mathrm{PB}, \mathrm{y}$ se forman 10 carteras de aproximadamente igual número de títulos. En cada año $t$ y en los años siguientes $t+\tau(\tau=1, \ldots, 5)$ disponibles se calcula la mediana de la rentabilidad financiera de las empresas incluidas en cada cartera. En las columnas figuran las medias calculadas para cada cartera y año con todas las observaciones medianas disponibles en cada caso, que oscila entre un máximo de 14 (para $t$ y $t+1$ ) y un mínimo de 10 (para $t+5)$. En la penúltima fila figura la media de los coeficientes de correlación entre los rangos del ratio PB mediano de las carteras en el año $t$ y de su ROE mediano en cada año $t+\tau$. En la última fila figuran esos mismos coeficientes calculados con las observaciones de los títulos individuales. La significación de los coeficientes de correlación se denota por:

* Con un nivel de confianza del $90 \%$.

$\because:$ Con un nivel de confianza del $95 \%$.

$\because:$ Con un nivel de confianza del $99 \%$. 
cada año, incluidas en la cartera 10, tienden a ser las firmas más rentables en ese año, y continúan siendo las empresas con una rentabilidad financiera más elevada hasta cinco años después por término medio. Por su parte, las empresas con una relación más reducida entre el valor de mercado y el valor contable de su patrimonio neto, incluidas en la cartera 1, muestran una rentabilidad financiera media en el año corriente considerablemente más reducida que la revelada por el resto de empresas, y tienden a seguir mostrando la rentabilidad más baja durante los cinco años siguientes.

Asimismo, se constata que la diferencia entre el ROE mediano de las carteras 10 y 1 decrece de forma monótona desde el año $t$ hasta el año $t+5$, pasando de un 23,6 a un $15 \%$. Esto indica que las rentabilidades financieras de las empresas con una relación más alta y más baja entre el valor bursátil y el valor contable de su patrimonio neto tienden a converger lentamente hacia un valor medio o central. Sin embargo, esta tendencia no es simétrica, y se manifiesta de manera más pronunciada con respecto a las empresas que muestran un ratio PB más bajo (cuyo ROE se incrementa en un $4,2 \%$ sobre el horizonte de cinco años), mientras que las empresas con un ratio PB más alto se caracterizan por un declive menor de su rentabilidad.

La correlación entre los rangos de las carteras según su ratio PB mediano en el año $t$ y según su ROE mediano en ese año y en los cinco años siguientes es significativamente positiva por término medio, no pudiendo ser rechazada la hipótesis $H_{1}$, y también muestra una tendencia decreciente a medida que se consideran años sucesivamente más alejados del año $t$. Sin embargo, cuando esa correlación se calcula utilizando los rangos de las observaciones correspondientes a los títulos individuales. se aprecia una reducción substancial de su magnitud en todos los años. Ello es previsible, puesto que la utilización de los valores medianos por carteras corrige el efecto de las observaciones extremas, aunque también supone una pérdida de información.

Una vez constatada la capacidad del ratio PB para predecir las diferencias (ordinales) en la rentabilidad financiera futura de las empresas, el siguiente paso es contrastar si los valores del ratio PB observados en cada año predicen las rentabilidades futuras mejor que la propia rentabilidad actual, como predice la hipótesis $H_{4}$. Para ello, llevamos a cabo la clasificación de las empresas en cada año del período 1986-1999 según su $R O E$, formando diez carteras de aproximadamente igual número de firmas. Para cada una de estas carteras determinamos su rentabilidad financiera mediana en los cinco años siguientes, repitiendo el procedimiento descrito para las carteras formadas según el ratio PB. 
La tabla 3 pone de manifiesto que, con relación a la muestra y período objeto de estudio, las empresas tienden a mantener a largo plazo su posición relativa de acuerdo con la rentabilidad obtenida sobre sus recursos propios en cada año. Esta persistencia de las rentabilidades resulta contradictoria con la evidencia previa hallada en el mercado norteamericano por Beaver [1970], Freeman et al. [1982] y Penman [1991], según la cual las rentabilidades financieras de magnitud extrema revierten en el futuro.

\section{TABLA 3}

RENTABILIDAD FINANCIERA FUTURA DE LAS CARTERAS FORMADAS SEGÚN LA DISTRIBUCIÓN DEL ROE EN CADA AÑO

\begin{tabular}{|c|r|r|r|r|r|c|}
\hline Cartera & $R O E_{t}$ & $R O E_{t+1}$ & $R O E_{t+2}$ & $R O E_{t+3}$ & $R O E_{t+4}$ & $R O E_{t+5}$ \\
\hline 1 & $-0,172$ & $-0,136$ & $-0,070$ & $-0,018$ & 0,049 & 0,053 \\
2 & $-0,030$ & $-0,009$ & 0,012 & 0,019 & 0,050 & 0,058 \\
3 & 0,037 & 0,047 & 0,040 & 0,068 & 0,083 & 0,121 \\
4 & 0,068 & 0,073 & 0,075 & 0,080 & 0,059 & 0,071 \\
5 & 0,095 & 0,107 & 0,105 & 0,112 & 0,126 & 0,109 \\
6 & 0,124 & 0,130 & 0,121 & 0,121 & 0,122 & 0,111 \\
7 & 0,156 & 0,154 & 0,149 & 0,147 & 0,132 & 0,130 \\
8 & 0,193 & 0,191 & 0,183 & 0,150 & 0,148 & 0,156 \\
9 & 0,261 & 0,218 & 0,204 & 0,170 & 0,124 & 0,168 \\
10 & 0,382 & 0,310 & 0,286 & 0,276 & 0,225 & 0,227 \\
\hline 9-1 & 0,554 & 0,446 & 0,356 & 0,294 & 0,176 & 0,174 \\
9-2 & 0,291 & 0,227 & 0,192 & 0,151 & 0,074 & 0,110 \\
\hline \multicolumn{2}{|c|}{ Correlación rangos: } & & & & & \\
Carteras & $0,976^{* * *}$ & $0,899^{* * *}$ & $0,756 * *$ & 0,559 & 0,518 \\
Títulos & $0,762^{* *}$ & $0,601^{* * *}$ & 0,486 & 0,378 & 0,351 \\
\hline
\end{tabular}

Al término de cada año $t(t=1986, \ldots, 1999)$ se ordenan laș empresas según su rentabilidad financiera, y se forman 10 carteras de aproximadamente igual número de títulos. En cada año $t$ y en los años siguientes $t+\tau(\tau=1, \ldots, 5)$ disponibles se calcula la mediana de la rentabilidad financiera de las empresas incluidas en cada cartera. En las columnas figuran las medias calculadas para cada cartera y año con todas las observaciones medianas disponibles en cada caso, que oscila entre un máximo de 14 (para $t$ y $t+1$ ) y un mínimo de 10 (para $t+5$ ). En la penúltima fila figura la media de los coeficientes de correlación entre los rangos del ROE mediano de las carteras en el año $t$ y en cada año $t+\tau$. En la última fila figuran esos mismos coeficientes calculados con las observaciones de los títulos individuales. La significáción de los coeficientes de correlación se denota por:

* Con un nivel de confianza del $90 \%$.

**: Con un nivel de confianza del $95 \%$.

**: Con un nivel de confianza del $99 \%$. 
En concreto, las empresas que revelan una tasa de rentabilidad sobre sus recursos propios más alta en cada año $t$, incluidas en la cartera 10 , continúan siendo en promedio las compañías más rentables hasta el año $t+5$. Por su parte, las empresas incluidas en la cartera 1 en el año $t$, que obtienen pérdidas en ese año, siguen mostrando una rentabilidad negativa durante los tres años siguientes $\mathrm{y}$, sobre los cinco años siguientes, tienden a ser las empresas menos rentables. Asimismo, la diferencia entre el ROE mediano de las carteras 10 y 1 en el año $t$ representa un 55,4\% en promedio, y cinco años después esa diferencia se ha reducido hasta un $17,4 \%$, y ello se debe en gran parte al incremento experimentado por la rentabilidad de las empresas inicialmente menos rentables. Por tanto, a pesar de la reversión en la magnitud de las rentabilidades extremas, la posición relativa de las empresas menos y más rentables inicialmente no se invierte.

La comparación de las tablas 2 y 3 pone de manifiesto que la magnitud de la correlación positiva entre las rentabilidades financieras observadas en los dos años siguientes al corriente con respecto a la rentabilidad actual es más acentuada que su correlación con respecto al ratio PB, utilizando tanto las observaciones medianas por carteras como las observaciones de los títulos individuales. Esto sugiere que, frente a lo que sería predecible si el mercado incorporase en el mecanismo de formación de precios la información no contenida en las cifras contables, la rentabilidad financiera calculada a partir de los estados financieros permite predecir las diferencias en las rentabilidades futuras a corto plazo mejor que los valores actuales del ratio $\mathrm{PB}$.

Para analizar con más detalle la diferencia entre la capacidad predictiva del ratio $\mathrm{PB}$ y del ROE, llevamos a cabo la agrupación de las empresas en cada año en tres carteras según su PB e, independientemente, en otras tres carteras según su ROE. La intersección de ambas clasificaciones resulta en nueve carteras compuestas por diferente número de títulos, con respecto a las cuales analizamos el comportamiento de sus rentabilidades futuras en la tabla 4.

Consistentemente con la correlación positiva hallada entre los valores actuales del ratio PB y del ROE, el análisis de esta tabla revela que las carteras de mayor tamaño son las que agrupan empresas con valores simultáneamente «altos» o «bajos» de ambas variables, mientras que las carteras que reúnen los títulos con un ratio $\mathrm{PB}$ «alto» («bajo») y un ROE «bajo» («alto») son las de menor tamaño. También es evidente que para las tres categorías de $\mathrm{ROE}$, las empresas con $\mathrm{PB}$ actual "alto" muestran un ROE futuro mayor que las empresas con un PB actual «bajo». Sin embargo, la diferencia entre el ROE observado en los años $t+1$ y $t+2$ para 


\section{TABLA 4}

RENTABILIDAD FINANCIERA FUTURA DE LAS CARTERAS FORMADAS SEGÚN LA DISTRIBUCIÓN DEL RATIO PB Y DEL ROE EN CADA AÑO

\begin{tabular}{|c|c|c|c|c|c|c|c|c|c|}
\hline \multicolumn{2}{|c|}{ Cartera } & \multirow{2}{*}{$N$} & \multirow{2}{*}{$P B$} & \multirow{2}{*}{$R O E_{1}$} & \multirow{2}{*}{$R O E_{t+1}$} & \multirow{2}{*}{$R O E_{t+2}$} & \multirow{2}{*}{$R O E_{t+3}$} & \multirow{2}{*}{$R O E_{l+4}$} & \multirow{2}{*}{$R O E_{t+5}$} \\
\hline$P B$ & $R O E$ & & & & & & & & \\
\hline Bajo & Bajo & 175 & 0,549 & $-0,017$ & $-0,022$ & $-0,014$ & 0,014 & 0,047 & 0,076 \\
\hline Bajo & Medio & 109 & 0,677 & 0,100 & 0,103 & 0,101 & 0,093 & 0,108 & 0,098 \\
\hline Bajo & Alto & 48 & 0,686 & 0,253 & 0,215 & 0,158 & 0,076 & 0,035 & 0,106 \\
\hline Medio & Bajo & 113 & 1,132 & 0,003 & 0,040 & 0,049 & 0,089 & 0,112 & 0,117 \\
\hline Medio & Medio & 129 & 1,156 & 0,115 & 0,110 & 0,108 & 0,117 & 0,109 & 0,091 \\
\hline Medio & Alto & 91 & 1,218 & 0,221 & 0,195 & 0,180 & 0,154 & 0,163 & 0,165 \\
\hline Alto & Bajo & 44 & 1,880 & $-0,057$ & 0,031 & 0,054 & 0,139 & 0,122 & 0,093 \\
\hline Alto & Medio & 95 & 2,046 & 0,124 & 0,143 & 0,144 & 0,151 & 0,145 & 0,131 \\
\hline Alto & Alto & 193 & 2,280 & 0,280 & 0,246 & 0,229 & 0,204 & 0,200 & 0,198 \\
\hline
\end{tabular}

Al término de cada año $t(t=1986, \ldots, 1999)$ se ordenan los títulos según su ratio PB y se forman 3 carteras de aproximadamente igual número de títulos (PB alto, medio y bajo). De manera independiente, los títulos también se ordenan segín su rentabilidad financiera y se forman otras 3 carteras (ROE alto, medio y bajo). La combinación de ambos clasificaciones resulta en 9 carteras, cuyo número total $(N)$ de títulos sobre los 14 años figura en la tercera columna. En cada año $t$ y en los años siguientes $t+\tau(\tau=1, \ldots, 5)$ disponibles se calcula la mediana de la rentabilidad financiera de las empresas incluidas en cada cartera. En las columnas figuran las medias calculadas para cada cartera y año con todas las observaciones medianas disponibles en cada caso, que oscila entre un máximo de 14 (para $t$ y $t+1$ ) y un mínimo de 10 (para $t+5)$.

las empresas con un ratio $\mathrm{PB}$ actual «alto» $\mathrm{y}$ «bajo» (con un ROE «bajo», «medio» o «alto») es muy inferior a la diferencia observada para las empresas con un ROE actual «alto» y «bajo» (y con un ratio PB «bajo», "medio» o "alto»). Por tanto, la rentabilidad actual permite predecir las rentabilidades mejor que el ratio PB sólo en el corto plazo.

La tabla 5 analiza la relación del ratio PB con el crecimiento futuro de los recursos propios, siguiendo para su construcción un procedimiento similar al descrito para la tabla 1 . Conforme al modelo [1], la tasa de crecimiento para cada empresa y año $t+\tau$ se calcula como $g_{t+\mathrm{r}}=\left(B_{t+\tau} / B_{t}\right)-1$. 


\section{TABLA 5}

\section{CRECIMIENTO FUTURO DE LOS RECURSOS PROPIOS DE LAS CARTERAS FORMADAS SEGÚN LA DISTRIBUCIÓN DEL RATIO PB EN CADA AÑO}

\begin{tabular}{|c|c|c|c|c|c|}
\hline Cartera & $g_{t+1}$ & $g_{t+2}$ & $g_{i+3}$ & $g_{i+4}$ & $g_{t+5}$ \\
\hline 1 & $-0,070$ & $-0,159$ & $-0,229$ & $-0,423$ & $-0,422$ \\
2 & $-0,050$ & $-0,101$ & $-0,112$ & $-0,169$ & $-0,119$ \\
3 & $-0,013$ & $-0,045$ & $-0,062$ & $-0,034$ & $-0,121$ \\
4 & $-0,002$ & $-0,020$ & 0,019 & $-0,018$ & $-0,033$ \\
5 & 0,047 & 0,001 & $-0,021$ & $-0,004$ & $-0,125$ \\
6 & 0,005 & 0,084 & 0,117 & 0,090 & 0,116 \\
7 & 0,067 & 0,078 & 0,189 & 0,233 & 0,332 \\
8 & 0,064 & 0,115 & 0,135 & 0,181 & 0,183 \\
9 & 0,075 & 0,138 & 0,211 & 0,199 & 0,149 \\
10 & 0,114 & 0,130 & 0,173 & 0,201 & 0,110 \\
\hline $10-1$ & 0,183 & 0,289 & 0,402 & 0,624 & 0,532 \\
$9-2$ & 0,125 & 0,239 & 0,323 & 0,368 & 0,268 \\
\hline Corl. rangos: & & & & & \\
Carteras & $0,623 *$ & $0,634 *$ & $0,691 * *$ & $0,592 *$ & 0,330 \\
Títulos & 0,240 & 0,266 & 0,305 & 0,295 & 0,268 \\
\hline
\end{tabular}

Al término de cada año $t(t=1986, \ldots, 1999)$ se ordenan los títulos según su ratio $\mathrm{PB}, \mathrm{y}$ se forman 10 carteras de aproximadamente igual número de títulos. En cada año $t+\tau$ $(\tau=1, \ldots, 5)$ se calcula la mediana del crecimiento de los recursos propios (ajustado por splits) con respecto al año $t$ de las empresas incluidas en cada cartera. En las columnas figuran las medias calculadas para cada cartera y año con todas las observaciones medianas disponibles en cada caso, que oscila entre un máximo de 14 (para $t$ y $t+1$ ) y un mínimo de 10 (para $t+5$ ). En la penúltima fila figura la media de los coeficientes de correlación entre los rangos del ratio $\mathrm{PB}$ mediano de las carteras en el año $t$ y de su crecimiento mediano en cada año $t+\tau$. En la última fila figuran esos mismos coeficientes calculados con las observaciones de los títulos individuales. La significación de los coeficientes de correlación se denota por:

* Con un nivel de confianza del $90 \%$.

$\because$ Con un nivel de confianza del $95 \%$.

*w: Con un nivel de confianza del $99 \%$.

Del análisis de esta tabla se desprende que las empresas con un ratio PB más bajo en cada año se caracterizan por un decrecimiento sostenido de sus recursos propios en los cinco años siguientes. Por el contrario, las empresas con un ratio PB más alto en cada año sólo muestran la tasa de cre- 
cimiento más alta en el año siguiente. En conjunto, la correlación del ratio PB con el crecimiento futuro de los recursos propios no muestra un patrón decreciente similar al observado con respecto a la rentabilidad financiera futura, y su magnitud media también es generalmente más débil.

Por último, la tabla 6 analiza la relación del ratio PB con el coeficiente $\beta$. Para cada una de las carteras formadas según la distribución del ratio PB, ese coeficiente se estima por la pendiente del modelo de mercado:

$$
R_{p t}=\alpha_{p}+\beta_{p} R_{m t}+u_{p t}(t=\text { enero de } 1987, \ldots, \text { diciembre de 2000) }
$$

donde $R_{p t}$ es la rentabilidad de la cartera $p(p=1,2, \ldots, 10)$ en el mes $t$, y $R_{m t}$ es la rentabilidad del mercado en el mest, estimada por la media aritmética simple de las rentabilidades de todos los títulos disponibles en ese mes (4).

TABLA 6

RENTABILIDAD MEDIA Y RIESGO DE LAS CARTERAS FORMADAS SEGÚN LA DISTRIBUCIÓN DEL RATIO PB

\begin{tabular}{|c|c|c|c|c|}
\hline Cartera & $\begin{array}{c}P B \\
\text { medio }\end{array}$ & $\begin{array}{c}\text { Rentabilidad } \\
\text { mensual media }\end{array}$ & $\begin{array}{c}\text { Desviación } \\
\text { estándar }\end{array}$ & Beta \\
\hline 1 & 0,336 & 0,008 & 0,097 & 1,052 \\
2 & 0,569 & 0,017 & 0,104 & 1,325 \\
3 & 0,732 & 0,013 & 0,092 & 1,117 \\
4 & 0,881 & 0,014 & 0,087 & 1,088 \\
5 & 1,061 & 0,009 & 0,085 & 1,068 \\
6 & 1,232 & 0,011 & 0,085 & 1,068 \\
7 & 1,446 & 0,010 & 0,085 & 1,061 \\
8 & 1,734 & 0,003 & 0,076 & 0,941 \\
9 & 2,216 & 0,010 & 0,074 & 0,920 \\
10 & 3,639 & 0,007 & 0,079 & 0,936 \\
\hline $10-1$ & 3,302 & $-0,001$ & $-0,018$ & $-0,116$ \\
$9-2$ & 1,647 & $-0,007$ & $-0,030$ & $-0,405$ \\
\hline
\end{tabular}

Al término de cada año $t(t=1986, \ldots, 1999)$ se ordenan los títulos según su ratio $\mathrm{PB}, \mathrm{y}$ se forman 10 carteras de aproximadamente igual número de títulos. En la segunda columna figura la media del ratio PB para cada cartera y a lo largo de los 14 años. La tercera y cuarta columnas recogen la media y la desviación estándar computadas con la serie de rentabilidades mensuales observadas desde enero de 1987 hasta diciembre del 2000 para cada cartera. En la última columna figura la pendiente del modelo de mercado estimado con la serie de rentabilidades observadas para cada cartera.

(4) En la cartera de mercado se incluyen los títulos excluidos en la formación de las carteras-PB como, por ejemplo, los pertenecientes a las empresas financieras o con un ratio $\mathrm{PB}$ negativo. 
La tabla 6 confirma la existencia de una relación inversa entre el ratio PB y la $\beta$ a lo largo de las carteras descritas, consistentemente con la hipótesis $H_{3}$. Sin embargo, esa relación no es monótona y las empresas con un ratio $\mathrm{PB}$ más bajo muestran un coeficiente $\beta$ sólo ligeramente superior a la unidad, mientras que las empresas un ratio PB más alto muestran el segundo coeficiente más bajo. Como consecuencia, la $\beta$ de la cartera 1 sólo es débilmente superior a la $\beta$ de la cartera 10 , lo cual es consistente con la escasa diferencia entre la rentabilidad mensual media de ambas carteras (igual a un $0,1 \%$ ). Por el contrario, entre las carteras 2 y 9 , que agrupan las empresas con un ratio $\mathrm{PB}$ bajo y alto aunque no extremo, si se aprecia una tendencia monótona decreciente de la $\beta$, y la diferencia entre los coeficientes estimados para ambas carteras triplica la observada para las carteras 1 y 10.

\subsection{ANÁLISIS DE REGRESIÓN}

El análisis previo únicamente contempla la relación bivariante del ratio PB con cada uno de sus determinantes fundamentales, utilizando los valores medianos por carteras. Asimismo, con respecto a la relación del ratio $\mathrm{PB}$ con la rentabilidad financiera y el crecimiento de los recursos propios, se analiza entre el año $t$ y cada año futuro $t+\tau$. Con el análisis de regresión que realizamos a continuación tratamos de paliar tales limitaciones. En concreto, nuestro objetivo es determinar en que medida la variación entre las observaciones del ratio $\mathrm{PB}$ de los títulos individuales (en cada año y sobre el período analizado) se puede explicar como una combinación (lineal) de sus tres determinantes fundamentales que predice el modelo [1].

No obstante, en primer lugar evaluamos la relación del ratio PB con cada uno de los tres factores del modelo [1] mediante la estimación del siguiente modelo de regresión simple:

$$
P B_{i t}=\gamma_{0}+\gamma_{1} Z_{i t}+e_{i t}
$$

donde $Z_{i t}$ es igual a: (i) la media geométrica de las rentabilidades financieras observadas para cada empresa $i$ desde el año $t+1$ hasta el año $t+5\left(R_{\left.O E L P_{i}\right)}\right)$; (ii) la media geométrica del crecimiento de los recursos propios observado para cada empresa $i$ desde el año $t+1$ hasta el año $t+4\left(G L P_{i t}=\left(B_{i, t+4} / B_{i t}\right)^{1 / 4}-1\right)$, y (iii) el coeficiente $\beta$ del título $i$ en el año $t$. Para la estimación el coeficiente $\beta$, asignamos a cada título $i$ y año $t$ la $\beta$ que el modelo [2] predice para la cartera-PB en la que resulta incluido al término de ese año. De esta forma, la $\beta$ de un título puede cambiar de un 
año a otro si cambia su emplazamiento de una cartera a otra (en función de cual sea su ratio $\mathrm{PB}$ ) (5).

Los resultados de la estimación del modelo [3] sobre el panel de observaciones empresa-año disponibles sobre el período 1986-1999 figuran en la primera parte de la tabla 7. Resulta evidente que la relación del ratio PB con cada uno de sus determinantes fundamentales es estadísticamente significativa y consistente con las hipótesis de partida. Sin embargo, la variable crecimiento deja de ser significativa cuando se incluye la rentabilidad futura o la $\beta$ como variable explicativa adicional en el modelo [3].

La pérdida de la capacidad explicativa del crecimiento, puede obedecer a la elevada correlación lineal de $G L P_{i t}$ con $R O E L P_{i t}$ y $\beta_{i t}$, como sugiere el hecho de que el estadístico $F$ permite rechazar la hipótesis de que los coeficientes de regresión asociados a $G L P_{i t}$ y a la otra variable son simultáneamente iguales a cero (6). Para contrastar la hipótesis $\mathrm{H}_{2}$, y determinar si el crecimiento ejerce realmente un efecto palanca sobre la rentabilidad futura, estimamos el siguiente modelo:

$$
\begin{gathered}
P B_{i t}=\gamma_{0}+\gamma_{1} D G L P_{i t}+\gamma_{2} R O E L P_{i t}+\gamma_{3} \beta+ \\
\gamma_{4} R O E L P_{i t} * D G L P_{i t}+\gamma_{5} \beta_{i t}{ }^{*} D G L P+e_{i t}
\end{gathered}
$$

donde $D G L P_{i t}$ es una variable que toma valor 1 si $G L P_{i t}$ es mayor que la mediana del mercado en el año $t$, y valor 0 en otro caso. Por tanto, $\gamma_{2}$ mide la relación del ratio $\mathrm{PB}$ con la rentabilidad futura para la submuestra de empresas caracterizadas por un crecimiento futuro de sus recursos propios bajo, mientras que $\gamma_{4}$ cuantifica el efecto incremental de la rentabilidad futura sobre el ratio PB cuando el crecimiento futuro es alto. Así pues, la hipótesis $H_{2}$ predice que el coeficiente $\gamma_{4}$ será positivo y significativamente distinto de cero.

El segundo panel de la tabla 7 refleja que cuando la ecuación [4] se estima sin incluir la $\beta$, el efecto de la rentabilidad futura sobre el ratio PB es significativamente superior cuando el crecimiento futuro es alto, consistentemente con la hipótesis $H_{2}$. En concreto, nuestros resultados indican que, en promedio, un incremento en un $10 \%$ de rentabilidad futura

(5) Alternativamente, otros estudios estiman secuencialmente la $\beta$ de cada título utilizando sus rentabilidades mensuales pasadas (sobre un horizonte que oscila entre 36 y 60 meses). Sin embargo, este procedimiento reduciría considerablemente el tamaño muestral, al no poder contar con los títulos que permanecen en la muestra menos de tres años y eliminar (como mínimo) los tres primeros años disponibles para el resto de empresas, introduciendo un sesgo de supervivencia.

(6) La pendiente de la regresión de $R O E L P_{i t}$ sobre $G L P_{i t}$ es igual a $0,599(t=8,495)$, y la pendiente de $R O E L P_{i t}$ sobre $\beta_{i t}$ es igual a $-0,115(t=-4,087)$. 
TABLA 7

\section{REGRESIÓN DEL RATIO PB SOBRE SUS DETERMINANTES FUNDAMENTALES (PANEL DE OBSERVACIONES EMPRESA-AÑO DEL PERÍODO 1986-1999)}

\begin{tabular}{|c|c|c|c|c|c|c|c|c|}
\hline \multicolumn{9}{|c|}{$P B_{i t}=\gamma_{0}+\gamma_{1} R O E L P_{i t}+\gamma_{2} \beta_{i t}+\gamma_{3} G L P_{i t}+e_{i t}$} \\
\hline$\gamma_{0}$ & $\gamma_{1}$ & $\gamma_{2}$ & $\gamma_{3}$ & & & $N$ & $R^{2}$ ajust. & $F$ \\
\hline $\begin{array}{l}1,096^{* * * *} \\
(22,512)\end{array}$ & $\begin{array}{c}2,433^{1 * * *} \\
(6,412)\end{array}$ & & & & & 548 & 0,190 & $129,445^{* * * * *}$ \\
\hline $\begin{array}{l}7,508^{* * * * 4} \\
(24,444)\end{array}$ & $(-20,882)$ & $-5,779 \div \div$ & & & & 997 & 0,351 & $539,065^{* * * * 4}$ \\
\hline $\begin{array}{l}1,342 \\
(35,053)\end{array}$ & $(3,471)$ & & $0,930^{* * * * *}$ & & & 625 & 0,019 & $13,488^{* * * t}$ \\
\hline $\begin{array}{l}6,079 * * * * \\
(18,676)\end{array}$ & $\begin{array}{c}1,483^{* * * x} \\
(4,325)\end{array}$ & $\begin{array}{l}-4,665^{* * * 4} \\
(-15,818)\end{array}$ & & & & 548 & 0,447 & $222,386^{* * * * *}$ \\
\hline $\begin{array}{l}1,064^{\prime 2}=\frac{1}{n-1} \\
(18,775)\end{array}$ & $\begin{array}{c}2,515^{* *-1} \\
(5,198)\end{array}$ & & $\begin{array}{c}-0,355 \\
(-0,889)\end{array}$ & & & 515 & 0,187 & $60,249 \cdots \cdots$ \\
\hline $\begin{array}{l}6,885^{* * * *} \\
(17,989)\end{array}$ & & $\begin{array}{r}-5,280^{* * * * *} \\
(-15,157)\end{array}$ & $\begin{array}{c}0,322 \\
(1,392)\end{array}$ & & & 625 & 0,346 & $166,468^{* * * * *}$ \\
\hline \multicolumn{9}{|c|}{$P B_{i t}=\gamma_{0}+\gamma_{1} D G L P_{i t}+\gamma_{2} R O E L P_{i t}+\gamma_{3} \beta+\gamma_{4} R O E L P_{i t} * D G L P_{i t}+\gamma_{3} \beta_{i t} * D G L P+e_{i t}$} \\
\hline$\gamma_{0}$ & $\gamma_{1}$ & $\gamma_{2}$ & $\gamma_{3}$ & $\gamma_{4}$ & $\gamma_{5}$ & $N$ & $R^{2}$ ajust. & $F$ \\
\hline $\begin{array}{l}1,090^{* * * \pi} \\
(20,088)\end{array}$ & $\begin{array}{c}-0,155 \\
(-1,333)\end{array}$ & $\begin{array}{c}2,085^{* \ldots * *: *} \\
(3,925)\end{array}$ & & $\begin{array}{l}1,321^{*} \\
(1,591)\end{array}$ & & 515 & 0,192 & $41,822^{* * *}$ \\
\hline $\begin{array}{l}5,815^{* * * *} \\
(12,097)\end{array}$ & $\begin{array}{c}0,091 \\
(0,128)\end{array}$ & $\begin{array}{c}1,476^{\text {th: }} \\
(3,234)\end{array}$ & $\begin{array}{l}-4,380^{*}: k \\
(-10,186)\end{array}$ & $\begin{array}{c}0,540 \\
(0,607)\end{array}$ & $\begin{array}{c}-0,242 \\
(-0,391)\end{array}$ & 515 & 0,438 & $81,049 * * 2$ \\
\hline
\end{tabular}

$P B_{i}$ es la relación entre el precio y el valor contable por acción (ajustado por splits) observada para la empresa $i$ al término del ejercicio cerrado en el año $t . R O E L P_{i t}$ es la media geométrica de las rentabilidades financieras observadas para cada empresa $i$ desde el año $t+1$ hasta el año $t+5$. GLP $_{\text {it }}$ es la media geométrica del crecimiento de los recursos propios (ajustado por splits) observado para cada empresa $i$ desde el año $t+1$ hasta el año $t+4$. $\beta$ es el riesgo de mercado del título $i$ en el año $t$, para cuya medida se utiliza la pendiente del modelo de mercado estimado con la serie de rentabilidades mensuales observadas desde enero de 1987 hasta diciembre del 2000 para cada una de las 10 carteras formadas según la distribución del ratio $\mathrm{PB}$ al término de los años 1986-1999 (que figuran en la última columna de la tabla 6), asignando esas estimaciones a los títulos incluidos en cada cartera y año. $D G L P_{i n}$ es una variable que toma valor 1 si $G L P_{i t}$ es mayor que la mediana del mercado, y valor 0 en otro caso. $N$ es el número de observaciones empresa-año utilizadas en la estimación de los modelos. Debajo de cada coeficiente figura el valor del estadístico $t$ ajustado según el procedimiento de White [1980]. La significación de los estadísticos $t$ y $F$ (que figura en la última columna) se denota por:

* Con un nivel de confianza del $90 \%$.

:*: Con un nivel de confianza del $95 \%$.

$\because:$ Con un nivel de confianza del $99 \%$. 
motiva un aumento del ratio $\mathrm{PB}$ de 0,208 si el crecimiento futuro es bajo $\left(D G L P_{i t}=0\right)$ y de 0,341 si el crecimiento futuro es alto $\left(D G L P_{i t}=1\right)$. Sin embargo, es preciso notar que cuando se estima la regresión [4], la inclusión de la $\beta$ motiva que el coeficiente $\gamma_{4}$, deje de ser significativamente distinto de cero aunque continúe siendo positivo. Ello puede obedecer nuevamente a la colinealidad entre las variables explicativas, dado que el estadístico $F$ no permite rechazar la especificación del modelo [4].

Por último, la contrastación de la hipótesis $H_{4}$ se llevó a cabo estimando el siguiente modelo:

$$
R O E_{i t+\tau}=\gamma_{0}+\gamma_{1} Z_{i t}+e_{i t} \quad(\tau=1,2, \ldots, 5)
$$

y los resultados obtenidos para $Z_{i t}=R O E_{i t}$ y $Z_{i t}=P B_{i t}$ sobre el panel de observaciones del período 1986-1999 figuran en los dos paneles de la tabla 8 .

Cuando la ecuación [5] se estima incluyendo como variable dependiente la rentabilidad financiera a un año $\left(R O E_{i t+1}\right)$ y como variable independiente la rentabilidad financiera actual $\left(Z_{i t}=R O E_{i t}\right)$, la pendiente $\gamma_{1}$ es significativamente distinta de cero $(t=15,663)$ y de uno $(t=-5,262)$. Por tanto, las rentabilidades financieras no se ajustan a un proceso de reversión a la media (en cuyo caso $\gamma_{1}=0$ ), pero tampoco son las realizaciones de un recorrido aleatorio en sentido estricto (lo que equivaldría a $\gamma_{1}=1$ ).

Por otra parte, un análisis de los coeficientes de determinación pone de manifiesto su tendencia decreciente a medida que la variable dependiente es la rentabilidad financiera observada en años sucesivamente más alejados del año actual $t$. Asimismo, el examen de los coeficientes $R^{2}$ de ambos paneles confirma la evidencia previamente hallada utilizando el análisis de correlación. En concreto, la rentabilidad financiera actual permite explicar una proporción mayor de la varianza de las rentabilidades futuras a corto plazo (en los años $t+1$ y $t+2$ ) que el ratio $\mathrm{PB}$ actual. En cambio, a partir del año $t+3$, el ratio PB explica una fracción de la variación de la rentabilidad financiera que, aunque reducida, es mayor que la explicada por el ROE actual. 
TABLA 8

REGRESIÓN DE LAS RENTABILIDADES FINANCIERAS FUTURAS SOBRE LOS VALORES ACTUALES DE LA RENTABILIDAD Y EL RATIO PB (PANEL DE OBSERVACIONES EMPRESA-AÑO DEL PERÍODO 1986-1999)

$$
R O E_{i+\tau}=\gamma_{0}+\gamma_{1} Z_{i i}+e_{i t}
$$

\begin{tabular}{|c|c|c|c|}
\hline & $\gamma_{0}$ & $\gamma_{1}$ & $R^{2}$ ajust. \\
\hline \multicolumn{4}{|l|}{$Z_{i t}=R O E_{i t}$} \\
\hline$R O E_{i t+1}$ & $\begin{array}{c}0,023 * * * * \\
(2,822)\end{array}$ & $\begin{array}{l}0,748 * * * \\
(15,663)\end{array}$ & 0,507 \\
\hline$R O E_{i t+2}$ & $\begin{array}{c}0,046^{* * t ; *} \\
(4,463)\end{array}$ & $\begin{array}{c}0,554^{* * *} \\
(9,162)\end{array}$ & 0,241 \\
\hline$R O E_{i t+3}$ & $\begin{array}{c}0,071 * * * * \\
(7,391)\end{array}$ & $\begin{array}{c}0,378 * * * \\
(6,022)\end{array}$ & 0,113 \\
\hline$R O E_{i t+4}$ & $\begin{array}{c}0,096^{* * * *} \\
(9,033)\end{array}$ & $\begin{array}{c}0,221 * \cdots * \\
(2,981)\end{array}$ & 0,039 \\
\hline$R O E_{i t+5}$ & $\begin{array}{l}0,106^{* * * *} \\
(10,217) \\
\end{array}$ & $\begin{array}{c}0,197^{* \cdots} \\
(2,882) \\
\end{array}$ & 0,034 \\
\hline \multicolumn{4}{|l|}{$Z_{i t}=P B_{i t}$} \\
\hline$R O E_{i k+1}$ & $\begin{array}{c}0,005 \\
(0,441)\end{array}$ & $\begin{array}{l}0,076^{* * * x} \\
(11,930)\end{array}$ & 0,179 \\
\hline$R O E_{i t+2}$ & $\begin{array}{c}0,001 \\
(0,066)\end{array}$ & $\begin{array}{l}0,076 \\
(10,241)\end{array}$ & 0,165 \\
\hline$R O E_{i+3}$ & $\begin{array}{c}0,005 \\
(0,391)\end{array}$ & $\begin{array}{c}0,078 * * * \\
(8,348)\end{array}$ & 0,148 \\
\hline$R O E_{i+4}$ & $\begin{array}{l}0,033 * \% \\
(2,095)\end{array}$ & $\begin{array}{c}0,062^{* * * *} \\
(5,336)\end{array}$ & 0,085 \\
\hline$R O E_{i+5}$ & $\begin{array}{c}0,044^{* * * x} \\
(2,931)\end{array}$ & $\begin{array}{c}0,061^{* \cdots * x} \\
(5,773)\end{array}$ & 0,078 \\
\hline
\end{tabular}

$R O E_{i t}$ es la rentabilidad financiera, igual al cociente del resultado ordinario de la empresa $i$ en el ejercicio cerrado en el año $t$ entre el valor contable inicial de sus recursos propios. $P B_{i t}$ es la relación entre el precio y el valor contable por acción (ajustado por splits) observada para la empresa $i$ al término del ejercicio cerrado en el año $t$. Debajo de cada coeficiente figura el valor del estadístico $t$, ajustado según el procedimiento de White [1980], y su significación se denota por:

* Con un nivel de confianza del $90 \%$.

$*$ Con un nivel de confianza del $95 \%$.

**: Con un nivel de confianza del $99 \%$. 


\section{RESUMEN Y CONSIDERACIONES FINALES}

La teoría financiera predice que, en un mercado eficiente, el valor atribuido por los inversores al patrimonio neto de una empresa sobre su valor contable será una función de su capacidad de generación de resultados anormales, en exceso sobre la retribución de los recursos propios que compensa el riesgo soportado por los inversores. Por tanto, la creación de valor para los accionistas se conseguirá a través de: (i) el incremento de la rentabilidad obtenida por el patrimonio neto de la empresa sobre su coste, y (ii) el crecimiento de sus recursos propios. Estos dos factores (value drivers) motivan que la relación entre el valor de mercado y el valor contable de los recursos propios (el ratio $\mathrm{PB}$ ) sea una función positiva de las expectativas de rentabilidad financiera y crecimiento de los recursos propios, y una función negativa del coste de capital (determinado a su vez por el riesgo que soportan los accionistas).

El estudio empírico llevado a cabo analiza una muestra de empresas negociadas en el mercado de capitales español, para las que se dispone de las series de precios de sus acciones y las cuentas anuales de los ejercicios cerrados durante la totalidad o parte del período 1985-2000. En relación con esta muestra, nuestros resultados confirman la existencia de una relación positiva y robusta entre los valores contemporáneos del ratio $\mathrm{PB}$ y la rentabilidad financiera de las empresas. Al mismo tiempo, las rentabilidades financieras se caracterizan por una elevada persistencia, de manera que las empresas que obtienen una rentabilidad positiva y más alta (o negativa y más alta en valor absoluto) sobre su patrimonio neto en cada año tienden a mantener esa posición durante los años siguientes.

La persistencia que caracteriza a la rentabilidad financiera se traduce en una relación positiva entre el ratio PB observado en el año actual y las rentabilidades financieras futuras, que tiende a debilitarse a medida que se consideran años sucesivamente más remotos. Sin embargo, las rentabilidades observadas en cada año permiten predecir las rentabilidades a corto plazo mejor que el propio ratio $\mathrm{PB}$, mientras que a muy largo plazo el ratio PB es una variable predictiva mejor de las rentabilidades. Ello sugiere que el mercado refleja en los precios alguna información, no incorporada a las cifras contables, acerca la rentabilidad financiera futura. Por último, nuestros resultados también confirman la existencia de una relación positiva del ratio $\mathrm{PB}$ con el crecimiento futuro de los recursos 
propios, y negativa con respecto al riesgo medido por el coeficiente $\beta$ del modelo de mercado.

La evidencia aportada por nuestro estudio es susceptible de extensión en dos líneas:

- En primer lugar, si el modelo propuesto acerca de los determinantes del ratio PB proporciona un valor de equilibrio (o intrínseco) de ese ratio para cada empresa y año, el siguiente paso en la investigación consistiría en contrastar si las empresas que muestran un ratio PB muy desviado al alza (a la baja) de ese valor de equilibrio son sobrevaloradas (infravaloradas) por el mercado. En concreto, cabe contrastar si una estrategia de inversión en los títulos que muestran un ratio PB muy alto en comparación con su ROE, financiada con la venta de aquellos otros títulos caracterizados por un ratio $\mathrm{PB}$ muy bajo en relación con su ROE, permite realizar una rentabilidad anormal significativa.

- Alternativamente, puede ser interesante determinar si la variación observada del ratio PB que no es explicada por los factores analizados en este trabajo puede atribuirse a las diferencias en la política contable de las empresas. En particular, cabría contrastar si las empresas que muestran un ratio $\mathrm{PB}$ relativamente más alto se caracterizan por la utilización de procedimientos contables que se traducen en un valoración más conservadora de sus recursos propios.

\section{REFERENCIAS BIBLIOGRÁFICAS}

BEAVER, W. [1970]: «The time series behavior of earnings», Joumal of Accounting Research, vol. 8 (suplemento), pp. 62-99.

Beaver, W., y Ryan, S. [2000]: "Biases and lags in book value and their effects on the ability of the book-to-market ratio to predict book return on equity», Joumal of Accounting Research, vol. 38, pp. 127-148.

BERNARD, V. [1994]: «Accounting-based valuation methods, determinants of market-to-book ratios, and implications for financial statements analysis», documento de trabajo, Universidad de Michigan.

CAPAUL, C.; ROWLEY, I., y SHARPE, W. [1993]: «International value and growth stock returns», Financial Analysts Joumal, enero-febrero, pp. 27-36.

CHAN, A., y ChuI, A. [1996]: «An empirical re-examination of the cross-sectional of expected returns: UK evidence», Joumnal of Business, Finance and Accounting, vol. 23, pp. 1435-1452.

CHAN, L.; HAMAO, Y., y LAKONISHOK, J. [1991]: «Fundamentals and stock returns in Japan’», Joumnal of Finance, vol. 46, pp. 1739-1764. 
Chan, L.; JegadeEsh, N., y LAKONISHOK, J. [1995]: «Evaluating the performance of value versus glamour stocks: The impact of selection bias", Joumal of Financial Economics, vol. 38, pp. 269-296.

Damodaran, A. [1996]: Investment valuation, John Wiley \& Sons, Nueva York.

DAvis, J. [1994]: "The cross-section of realized stock returns: The pre-COMPUSTAT evidence", Journal of Finance, vol. 49, pp. 1579-1593.

FAMA, E., y FRENCH, K. [1992]: «The cross-section of expected returns», Joumal of Finance, vol. 47, pp. 427-465.

- [1995]: "Size and book-to-market factors in earnings and returns», Joumal of Finance, vol. 50, pp. 131-155.

- [1996]: «Multifactor explanations of asset pricing anomalies», Joumal of Finance, vol. 51, pp. 55-84.

- [1998]: "Value versus growth: International evidence», Joumal of Finance, vol. 53, pp. 1975-1998.

FAIRIFIELD, P., y HaRRIS, T. [1993]: «Price-earnings and price-to-book anomalies: Tests of an intrinsec value explanation", Contemporary Accounting Research, vol. 9, pp. 590-611.

Freeman, R.; Ohlson, J., y Penman, S. [1982]: «Book rate of return and prediction of earnings changes: An empirical investigation, Joumal of Accounting Research, vol. 20, pp. 639-653.

JENSEN, G.; JoHNSON, R., y MERCER, J. [1997]: "New evidence on size and price-tobook effects in stock returns», Financial Analyst Joumal, noviembre-diciembre, pp. 34-42.

Kothari, S.; Shanken, J., y SloAN, R. [1995]: "Another look at the cross-section of expected stock returns", Joumal of Finance, vol. 50, pp. 185-224.

La Porta, R.; Lakonishok, J.; Shleifer, A., y Vishny, R. [1997]: "Goods news for value stocks: Further evidence on market efficiency", Journal of Finance, vol. 52, pp. 859-874.

LAKONISHOK, J.; SHLEIFER, A., y VISHNY, R. [1994]: "Contrarian investment, extrapolation, and risk», Journal of Finance, vol. 49, pp. 1541-1578.

LEV, B., y Sougiannis, T. [1999]: «Penetrating the book-to-market black box: The R\&D effect", Joumal of Business, Finance and Accounting, vol. 26, pp. 419-449.

OHLSON, J. [1995]: «Earnings, book values and dividends in equity valuation», Contemporary Accounting Research, vol. 11, pp. 661-687.

PENMAN, S. [1991]: "An evaluation of accounting rate-of-return", Joumal of Accounting, Auditing and Finance, vol. 6, pp. 233-255.

- [1996]: "The articulation of price-earnings ratios and market-to-book ratios and the evaluation of growth", Joumal of Accounting Research, vol. 34, pp. 235-259.

Rosenberg, B.; ReID, K., y LANSTEIN, R. [1985]: "Persuasive evidence of market inefficiency", Joumzal of Portfolio Management, vol. 11, pp. 9-17.

RuEDA, J. [2001]: "Identificación de oportunidades de inversion utilizando las cifras constables de resultados y recursos propios», documento de trabajo, Universidad de Sevilla. 
RYAN, S. [1995]: «A model of accrual measurement with implications for the evolution of the market-to-book ratio», Joumal of Accounting Research, vol. 33, pp. 95-112.

WILCOX, J. [1984]: «The P/B-ROE valuation model», Financial Analysts Joumal, enero-febrero, pp. 58-66. 